\title{
Aula Inversa: una Oportunidad para el Desarrollo de Competencias Transversales
}

\section{Inmaculada C. Fita ${ }^{a}$, Jose F. Monserrat ${ }^{b}$ y Germán Moltóc}

infifer@fis.upv.es ETSIAMN. Universitat Politècnica de València , jomondel@upv.es EPSG. Universitat Politècnica de València y ${ }^{c}$ gmolto@dsic.upv.es ETSINF. Universitat Politècnica de València

\begin{abstract}
A preliminary work about the impact of the Flipped Classroom $(F C)$ in the development of different Soft Skills (SS) defined by the UPV is shown in this article. Throughout the 2015-16 academic year, some particular subjects in the Universitat Politècnica de València (UPV) are checkpoints to monitor the implementation and assessment of the specific SS. All SS must be developed every year and their control is distributed for assessment in the different courses to ensure a complete evaluation of the SS is offered at the several training stages students. The professors belonging to the MATI EICE group have previous experience in making digital materials and using them in the FC methodology. Also, due to the fact that we are a multidisciplinary group, each member has assessed the SS for which their subjects were control points, which enriches this study, offering a broad overview regarding the possibilities to use FC in the classroom to work the skills the students must have at the end of their education.
\end{abstract}

Keywords: Flipped Teaching, Soft Skills, Methodology

\section{Resumen}

En este trabajo se presenta un estudio preliminar del impacto de la técnica de Aula Inversa (AI) en el desarrollo de diferentes Competencias Transversales (CT). En el curso 15-16 todas las asignaturas son puntos de control en el desarrollo de alguna de las CT que define la Universitat Politècnica de València (UPV). Todas las CT han de ser desarrolladas en todos los cursos del grado y han de ser evaluadas en las distintas asignaturas con el fin de ofrecer una evaluación completa de competencias en distintas etapas de la formación de cada estudiante. Nuestro grupo EICE de profesores (MATI) tiene experiencia previa en la preparación de materiales digitales y su aplicación con la técnica de Aula Inversa. Al ser un grupo multidisciplinar a cada miembro del grupo le corresponde evaluar una competencia distinta, lo cual enriquece el estudio, ofreciendo una visión bastante amplia acerca de las posibilidades de aplicación.

Palabras clave: Aula Inversa, Competencias Transversales, metodología. 


\section{Introducción}

La educación universitaria, con un reconocido prestigio alcanzado tras más de un milenio de historia, está inmersa en un profundo proceso de renovación. Renovarse o morir, sería el espíritu que caracteriza a las instituciones universitarias en todo el mundo. Las amenazas son muchas, pero sin duda la sociedad del conocimiento, conectada en cualquier situación a la más abundante y organizada fuente de información jamás conocida, ya no necesita adquirir en sus individuos piezas de conocimiento específico, sino más bien consolidar capacidades holísticas de procesado de la información, síntesis y toma eficaz de decisiones.

Además, dado que existe gran cantidad de material online en la red, muchos cursos presenciales han incorporado estos materiales en sus repositorios, convirtiéndose así en verdaderos cursos semipresenciales, y ofreciendo al alumno la posibilidad de diversificar su aprendizaje [1]. En esta situación se puede dar la circunstancia que los alumnos no consideren interesante la clase presencial, preparando las asignaturas con el material online, sin apenas ir a clase. Para evitar esta circunstancia, las clases presenciales cada vez más tienen que aportar un valor añadido a los cursos no presenciales.

En este contexto, la clase magistral como único elemento docente pierde todo sentido, más bien será necesario que los alumnos tengan un papel más proactivo en su proceso de aprendizaje. En esta dirección, y desde 2007, la comunidad universitaria está cambiando el enfoque educativo en lo que se conoce como Aula Inversa (AI), o Flipped Classroom (FC) en inglés. El Aula Inversa es un enfoque educativo en el que el aprendizaje directo se traslada hacia el espacio individual, y el espacio colectivo en el aula se sustituye por un ambiente de aprendizaje dinámico e interactivo, en el que el rol de profesor pasa a ser más el de mentor y dinamizador del aprendizaje creativo y grupal [2].

El método de AI brinda la oportunidad de utilizar las TICs a favor del profesor, permitiendo preparar con anterioridad las clases presenciales, de modo que en cada clase el alumno experimente e interaccione con sus compañeros y con el profesor, multiplicando las posibilidades de aprendizaje en el aula. Cuando el método esté implementado, el alumno será consciente de los resultados de su aprendizaje y será un agente activo de su propio aprendizaje. El alumno reconocerá la importancia de las actividades presenciales y el profesor se convertirá en el mejor de los activos de la universidad.

Además, esta revolución en las aulas tiene que ir acompañada de un cambio también importante en la forma de evaluar a los alumnos. Parece cada vez menos relevante saber si el alumno ha memorizado ciertos conceptos, sino más bien evaluar si es competente en un conjunto de destrezas fundamentales para su profesión [3]. Por ello es interesante preparar aplicar la metodología de aula inversa en las actividades más prácticas del curriculum: seminarios y prácticas [4]. En esta dirección, la Universitat Politècnica de València, se ha marcado como objetivo para el curso 2015-2016 asignar la evaluación de Competencias Transversales (CT) en un conjunto de asignaturas que funcionarán como puntos de control [5]. A partir de aquí se podrá determinar hasta qué punto un alumno ha adquirido esas competencias que se le suponen al acabar sus estudios.

(cc) EY-NC-ND 2016, Universitat Politècnica de València

Congreso IN-RED (2016) 


\section{Objetivos}

Este trabajo persigue como objetivo exponer y evaluar diferentes aproximaciones enmarcadas en lo que se conoce habitualmente como Aula Inversa pero aplicadas específicamente al desarrollo $\mathrm{y}$, en algunos casos, a la evaluación de competencias transversales.

El eje común de la experiencia radica en el uso de TIC (Tecnologías de la Información y de las Comunicaciones), como es el caso de material educativo audiovisual, típicamente videolecciones y video-ejercicios producidos íntegramente por los autores, como elemento conductor de las actividades de aula inversa y que posibilitan su aplicación al desarrollo de competencias transversales. También se utilizan otro tipo de herramientas tecnológicas que resultan de ayuda para la implementación del Aula Inversa.

\section{Desarrollo de la Innovación}

A continuación se exponen las diferentes experiencias desarrolladas y clasificadas por tipo de competencia, cuya descripción puede encontrarse en [5]:

\section{1. (CT01) Comprensión e integración}

En el curso 2014/2015 se evaluó la competencia sin realizar ninguna actividad específica para el desarrollo de la misma. Se evaluó dentro de los exámenes parciales de teoría problemas, como una pregunta de aplicación directa de algún concepto teórico. Generalmente la pregunta consistía en un pequeño cálculo sobre alguna ecuación física que permitiera comparar dos posibles estados de un sistema, normalmente se requería acompañar la solución con una valoración del resultado.

En el curso 2015/2016 se ha desarrollado una actividad específica para que el alumno pueda practicar la competencia, ésta se ha relacionado con una actividad dónde el alumno consigue bastante autonomía por desarrollarse bajo la técnica del aula inversa.

- Actividades desarrolladas relacionadas con la adquisición de la CT:

Preguntas y problemas que integran la teoría con las prácticas de laboratorio

- Descripción detallada de las actividades:

Cada práctica de laboratorio lleva asociada una batería de preguntas de respuesta múltiple sobre las que el alumno trabaja durante los 15 días en los que se desarrolla la práctica correspondiente. Estas preguntas están basadas en los modelos físicos que se comprueban en el laboratorio. Los alumnos pueden autoevaluar su competencia resolviendo las cuestiones en Exámenes de PoliformaT.

(c) EY-NC-ND 2016, Universitat Politècnica de València

Congreso In-Red (2016) 
- Criterios de evaluación:

Al finalizar las prácticas de laboratorio, junto con el examen de laboratorio, los alumnos realizan la evaluación de la competencia mediante un examen en PoliformaT de contenido similar a las baterías de cuestiones propuestas en las autoevaluaciones (Fig.1).

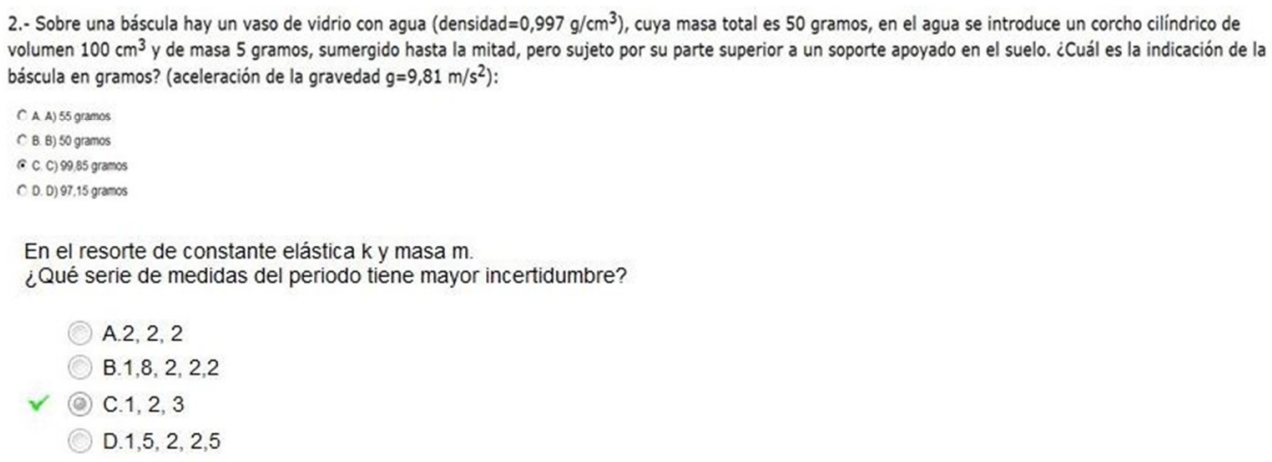

Fig. 1 Dos ejemplos de test de evaluación de la competencia, realizados en PoliformaT

- $\quad$ Actividad AI:

Los alumnos disponen de un vídeo que describe los aspectos técnicos más importantes de cada práctica. El libro de prácticas también es un material fundamental que los alumnos deben trabajar tanto en la sesión presencial como en el laboratorio. Además los alumnos deben responder un cuestionario de 5 preguntas relacionadas con el vídeo, antes de empezar la sesión presencial.

Al comienzo de cada sesión presencial los alumnos disponen de un espacio de 15 minutos para: resumir los objetivos de la práctica en un mapa conceptual, discutir aquellas preguntas y problemas propuestas en PoliformaT que hayan tenido mayor número de errores, así como resolver cualquier duda relacionada.

\section{2. (CT-13) Instrumental Específica}

En el Master Universitario en Computación Paralela y Distribuida (MUCPD), la asignatura Infraestructuras Avanzadas en Cloud (IAC) trabaja y es punto de control de las competencias transversales Instrumental Específica y Aprendizaje Permanente. En dicha asignatura, los alumnos aprenden diferentes plataformas de Cloud Computing, centrando el foco sobre Amazon Web Services (AWS), el principal proveedor de Cloud público. Se describen y se trabaja con los principales servicios de AWS para la creación de arquitecturas de aplicaciones escalables en la nube. Se trata de una asignatura eminentemente práctica en la que los alumnos deben experimentar con los múltiples servicios y utilizar las diferentes interfaces de acceso (línea de comandos, interfaz web) que les permita manejar los servicios de AWS para resolver problemas reales. 
Trabajar la competencia transversal Instrumental Específica resulta especialmente sencillo para este tipo de asignatura, que involucran el manejo de múltiples herramientas informáticas para, en este caso, combinar la funcionalidad de diferentes servicios para desplegar arquitecturas de aplicaciones en la nube.

Los alumnos requieren un entorno pre-configurado de trabajo que disponga de las herramientas software necesarias para llevar a cabo las actividades prácticas, así como las credenciales de usuario necesarias para utilizar los servicios de AWS. Para desplegar dicho entorno de prácticas se utilizó ODISEA [5] un sistema para definir entornos virtualizados computacionales de acuerdo a los requisitos hardware, software y de configuración necesarios para soportar la actividad educativa. En nuestro caso concreto se utiliza una máquina virtual configurada con múltiples cuentas de usuario con las credenciales específicas, la herramienta de línea de comandos de AWS y el material de prácticas (documentos, código fuente, etc.) necesario para llevar a cabo las prácticas. Usando ODISEA se despliega dicho laboratorio remoto en la nube (se utiliza de nuevo AWS) al que los alumnos se conectan utilizando SSH (una herramienta de línea de comandos) para llevar a cabo las actividades. También se conectan a la interfaz de consola web de AWS para gestionar los diferentes servicios.

La experiencia de Aula Inversa consistió en que los alumnos utilizasen el laboratorio remoto, disponible $24 \times 7$, para que los alumnos adquiriesen la destreza necesaria con las herramientas y servicios de AWS. Se proponía a los alumnos que iniciasen la realización de la actividad práctica en cualquier momento (incluso fuera del aula) para que pudiesen dedicar la sesión de aula también a progresar con las prácticas pero, sobre todo, para resolver las dudas surgidas durante la realización de las mismas).

Efectivamente, ofrecer un laboratorio remoto permite realizar las actividades prácticas en cualquier momento y no únicamente en el horario asignado. Esto facilita en gran medida que el alumno pueda progresar con las actividades prácticas y mantener el ritmo de la clase.

Esto contribuye a desarrollar la competencia transversal instrumental específica. Nótese que el despliegue de laboratorios remotos en la nube permite a los alumnos acceso ubicuo a los mismos, pudiendo conectarse a ellos en cualquier momento y desde cualquier lugar con acceso a Internet.

\section{3. (CT-11) Aprendizaje Permanente}

La asignatura IAC también es punto de control de la competencia transversal Aprendizaje Permanente. Al tratarse de una asignatura de Máster, procede considerar el nivel 3 de acuerdo a la clasificación mostrada en [5], que indica que el alumno debe desarrollar estrategias de aprendizaje propias para ampliar lo aprendido según sus necesidades personales y profesionales.

Para poder abordar el desarrollo de esta competencia transversal se procedió a desarrollar material educativo en formato online siempre accesible por el alumno desde el primer día. El material constaba de: vídeo-lecciones de unos 7-10 minutos de duración sobre los principales servicios de AWS; tests de auto-evaluación, que permiten al alumno ponerse a

(cc)) EY-NC-ND 2016, Universitat Politècnica de València

Congreso In-Red (2016) 
prueba tras cada unidad temática; un laboratorio computacional remoto, descrito en la sección anterior, que permite a los alumnos disponer de un entorno configurado para realizar las prácticas; guías de aprendizaje, que sugieren al alumno un itinerario de aprendizaje, si bien el alumno es libre de alterar dicho itinerario en base a sus propias necesidades personales y profesionales. Dicho material también se utiliza en el contexto de otras asignaturas de Cloud Computing impartidas en otras titulación y en el Curso Online de Cloud Computing con Amazon Web Services (AWS), ofertado a través del Centro de Formación Permanente.

La experiencia de Aula Inversa consiste por tanto en ofrecer dicha experiencia educativa desde el momento cero de la asignatura, posibilitando que el alumno tenga acceso a todo el material pero sugiriendo el profesor un ritmo de trabajo. El alumno puede ver las videolecciones en casa y realizar ciertas actividades prácticas en casa (o terminar aquellas prácticas que no ha podido finalizar durante las sesiones de aula) que son complementadas con el soporte dado por el profesor durante las sesiones de aula. Éstas combinan siempre una explicación teórica, complementaria a las vídeo-lecciones, aunque con cierto grado de solape para enfatizar los principales conceptos, seguida de tiempo libre para avanzar en las actividades prácticas.

El uso de este tipo de técnicas está muy relacionado con la formación online en sus múltiples variantes: cursos de formación online asíncrona, MOOCs, SPOCs, etc. Este tipo de técnicas favorece el desarrollo de la competencia transversal Aprendizaje Permanente.

\section{4. (CT10) Conocimiento de problemas contemporáneos}

Esta competencia hace referencia a la necesidad de que los estudiantes comprendan las cuestiones y valores políticos, sociales, legales y medioambientales contemporáneos, así como los mecanismos de expansión y difusión del conocimiento. Se trata de que desarrollen la capacidad de "estar al día" de los eventos actuales en su campo de conocimiento y en la sociedad en general.

Para trabajar esta competencia se tienen que buscar escenarios formativos en los que los alumnos dialoguen en profundidad este tipo de cuestiones, por lo que el AI se presenta como un elemento muy interesante para desarrollar esta competencia.

En el Grado en Ingeniería de Sistemas de Telecomunicación, Sonido e Imagen (GISTSI), la asignatura Sistemas de Comunicaciones Inalámbricas (SCI) trabaja y es punto de control de esta competencia. Se utilizó la técnica de AI, de manera que se ofrecieron varios documentos sobre seguridad electromagnética describiendo la posición oficial de la OMS, así como distintas webs dedicadas a inculcar temor acerca del peligro de la radiación móvil. Los alumnos leyeron y trabajaron el material en casa sin saber su posición al respecto. En clase se llevó a cabo una discusión pública por parejas del problema de la seguridad electromagnética, con pros y contras, realizándose después una valoración del argumento utilizado. El rol asignado al alumno fue aleatorio, de manera que se pretendía también desarrollar las capacidades de convicción.

(cc) EY-NC-ND 2016, Universitat Politècnica de València 
Para el desarrollo de los materiales se utilizó la aplicación Office Mix [7], que es un add-on gratuito de Office Power Point para la creación de contenidos específicos de AI. Gracias a esta aplicación, se pueden colgar los contenidos, incluir grabaciones de pantalla, encuestas (Fig.2), así como directamente la explicación del profesor, y todo ello desde el propio Office Power Point.

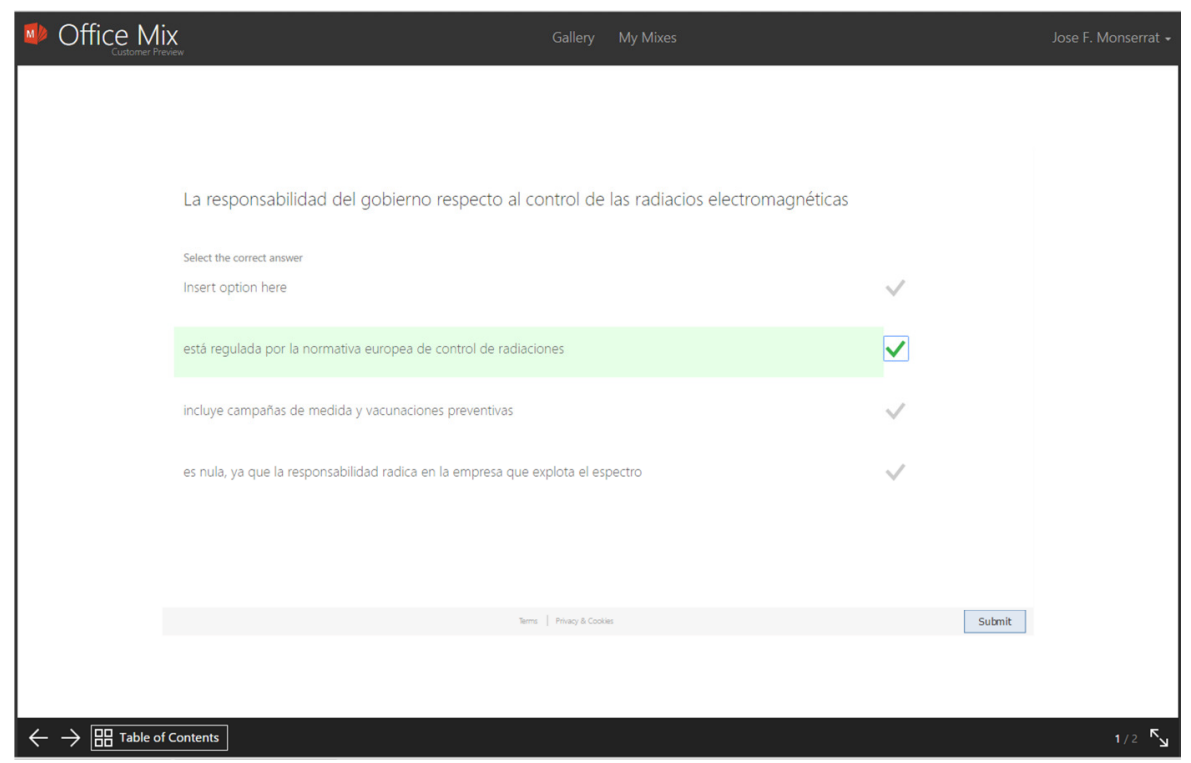

Fig. 2 Aspecto del resultado final de Office Mix para función del Aula Inversa.

\section{Resultados}

Los resultados también se exponen por competencias pues cada experiencia lleva un grado de desarrollo y en algunos casos no ha sido posible conseguir resultados todavía (asignaturas segundo semestre).

\section{1. (CT01) Comprensión e integración}

Esta experiencia se aplica a la asignatura de Fundamentos de Física en dos grados distintos: Ciencia y Tecnología de los Alimentos y Biotecnología. Se dispone de resultados del primero de los grados pues la asignatura se desarrolla en el primer semestre. A continuación se comparan los resultados obtenidos en dos cursos académicos 2014/2015 y 2015/2016.

En la Fig.3 se muestra un gráfico de barras dónde aparecen los resultados de la competencia en dos cursos académicos. La evaluación se realizó sobre 10, se asigna AExcelente $\epsilon$ [9-10], B-Adecuado $\epsilon$ [7-8,9], C-En desarrollo $\epsilon$ [5-6,9], D-No alcanzado $\epsilon$ [0$4,9]$. El porcentaje mostrado es el calculado por número de alumnos respecto de alumnos presentados. 


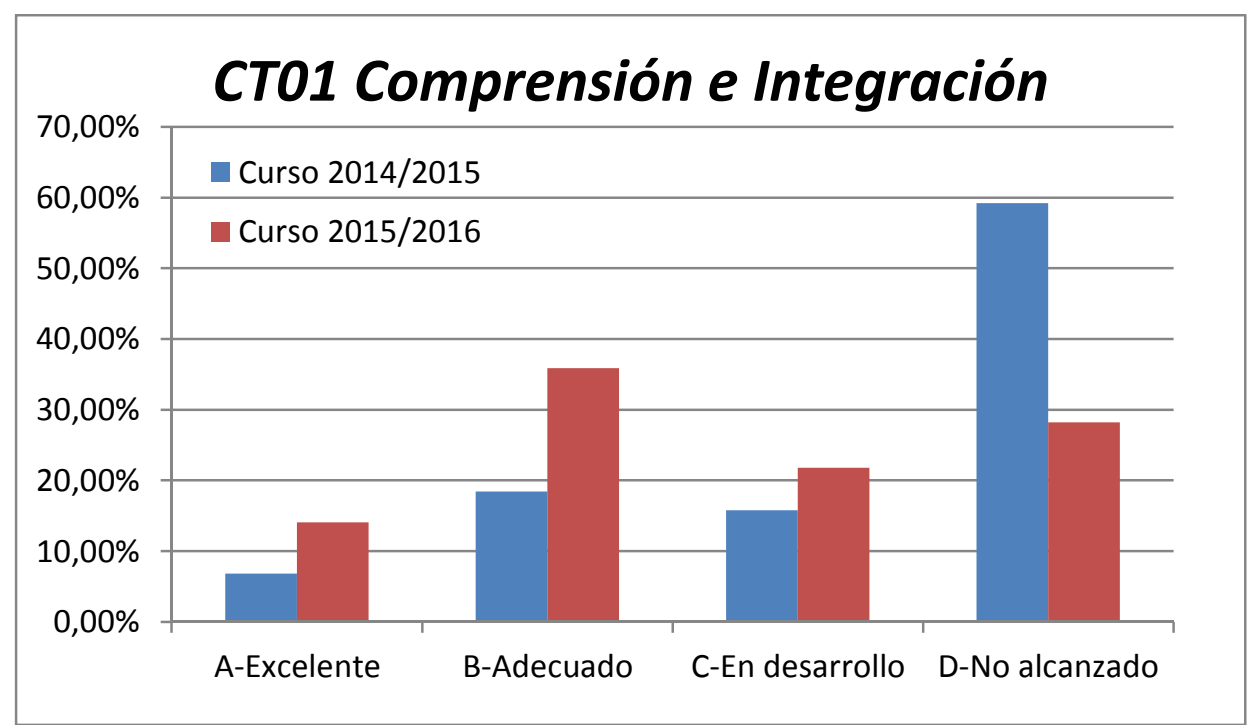

Fig. 3 Resultados de la CTO1 en la asignatura de Fundamentos Físicos para la Ciencia y Tecnología de los Alimentos en los cursos 2014/2015 y 2015/2016.

En la Fig.3 se observa que los resultados han cambiado completamente, si se analiza cada uno de los resultados por separado, encontramos que el porcentaje de alumnos con una evaluación A-Excelente ha pasado de 6,6\% a 14,1\%, en cuanto al grupo de B-Adecuado también se ha duplicado, el grupo C-En desarrollo también ha aumentado de 15,8\% a $21,8 \%$, aunque no de forma tan importante como el grupo D-No alcanzado que ha pasado de $59,2 \%$ a un $28,21 \%$.

\section{2. (CT-13) Instrumental Específica}

La Fig.4 muestra la valoración de los alumnos de IAC (y de otros alumnos que utilizan el mismo entorno de prácticas en otras asignaturas). Consideran que dicha herramienta es apropiada para llevar a cabo sus actividades. En conversaciones con ellos, valoran especialmente la disponibilidad $24 \times 7$ y la posibilidad de acceder al entorno de prácticas en cualquier momento.

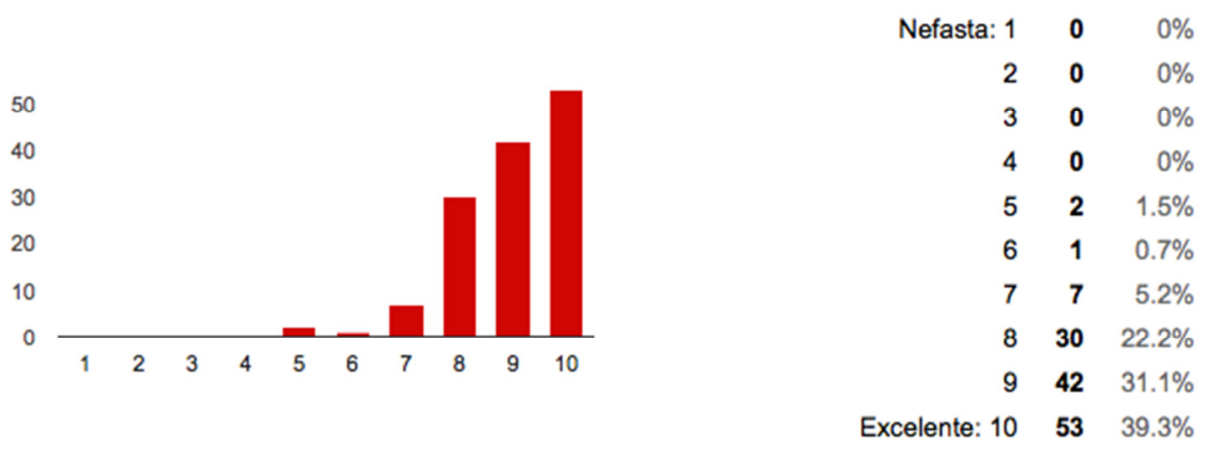

Fig. 4 Valoración del entorno de prácticas. 


\section{3. (CT-11) Aprendizaje Permanente}

La Fig.5 resume la valoración que hacen los alumnos sobre la calidad de las vídeolecciones. En general, valoran positivamente poder utilizar dicho material en cualquier momento y la posibilidad de utilizarlo como mecanismo alternativo cuando no pueden acudir a alguna clase de teoría.

\section{Valora la calidad de las vídeo-lecciones}

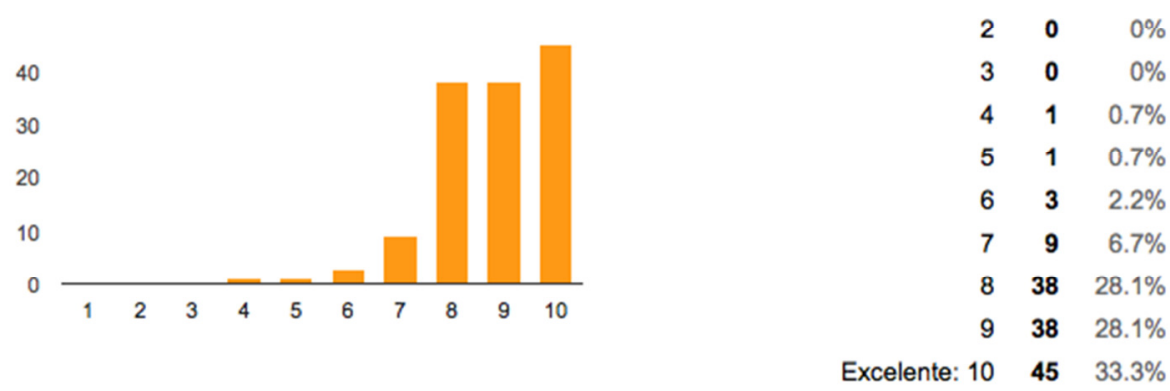

Fig.5 Valoración de la calidad de las vídeo-lecciones.

Por ultimo, la Fig.6 resume la valoración que hacen los alumnos de la satisfacción con las asignaturas en las que se utilizan las técnicas descritas en esta contribución. En general, los alumnos valoran positivamente poder disponer de material audio-visual, laboratorios remotos, test de auto-evaluación para poder ellos alterar el ritmo de aprendizaje y adaptarlo a sus necesidades profesionales y personales, aspecto destacado en la competencia transversal Aprendizaje Permanente.

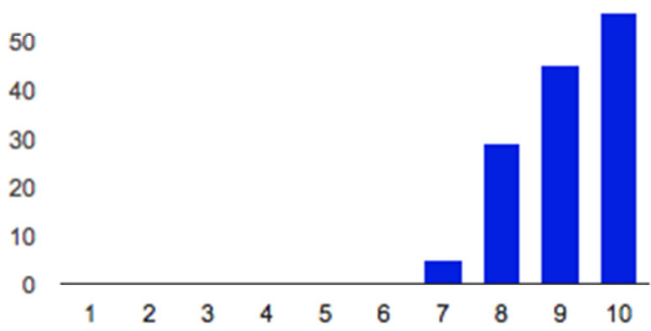

$\begin{array}{rrr}\text { No: } 1 & \mathbf{0} & 0 \% \\ \mathbf{2} & \mathbf{0} & 0 \% \\ 3 & \mathbf{0} & 0 \% \\ \mathbf{4} & \mathbf{0} & 0 \% \\ \mathbf{5} & \mathbf{0} & 0 \% \\ \mathbf{6} & \mathbf{0} & 0 \% \\ \mathbf{7} & \mathbf{5} & 3.7 \% \\ \mathbf{8} & \mathbf{2 9} & 21.5 \% \\ \mathbf{9} & \mathbf{4 5} & 33.3 \% \\ \text { Si: } 10 & \mathbf{5 6} & 41.5 \%\end{array}$

Fig.6 Valoración de la satisfacción con la asignatura.

\section{4. (CT10) Conocimiento de problemas contemporáneos}

Tras la realización de la actividad de discusión se realizaron dos encuestas, una relativa al aprendizaje de los conceptos (que recordemos que no se habían trabajado en clase) y otra respecto a la actividad en sí. Respecto a los resultados de aprendizaje (Fig.7), se observó un comportamiento mejor del esperado. Efectivamente parece que los alumnos que realizaron

(cc) EY-NC-No 2016, Universitat Politècnica de València

Congreso In-Red (2016) 
el trabajo en casa adquirieron los conocimientos esperados, y así el $61 \%$ de los alumnos obtuvo la máxima cualificación. Además, quedó patente en el alrededor de 10\% de suspensos, que la actividad de aula no fue suficiente para adquirir dicho conocimiento.

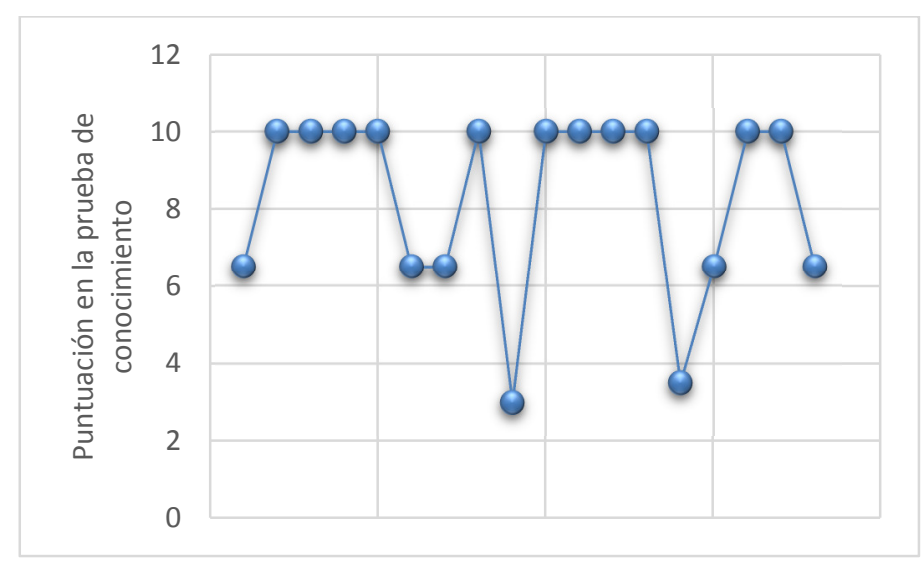

Fig.7 Notas obtenidas en la prueba de conceptos.

Por otro lado, la actividad en sí fue valorada como muy positiva por el $83 \%$ de los alumnos, mientras que en sólo un caso se marcó como decepcionante la actividad de AI.

\section{Conclusiones}

En la experiencia dirigida a la competencia CTO1 se muestra una mejora importante en los resultados obtenidos en este curso, lo cual demuestra la importancia de preparar actividades específicas para el desarrollo de la competencia. Evaluar el impacto que el AI ha tenido en esta experiencia es complicado, pues van vinculadas ambas actividades. Para conseguir un examen más profundo en la interacción de ambas se proponen algunas acciones para el curso próximo tales como desarrollar una actividad presencial específica de refuerzo, incentivar la realización de los test una vez finalizada la práctica y facilitar el desarrollo continuo de la competencia para una adquisición sólida.

Las competencias CT13 y CT11 se pueden trabajar principalmente mediante técnicas ampliamente utilizadas en la formación online, involucrando laboratorios remotos, vídeolecciones, test de auto-evaluación. Combinar este material para su uso en experiencias de AI es fácilmente aplicable a otras asignaturas que sean puntos de control de estas competencias, si bien requiere el esfuerzo extra de preparar material adicional.

(c) EY-NC-NO 2016, Universitat Politècnica de València 
Respecto a la competencia CT10, la aplicación del enfoque del AI fue un habilitador natural para su desarrollo. Consideramos que es imposible desarrollar la capacidad de comprensión de los problemas contemporáneos sin que el alumno se convierta en el protagonista de su aprendizaje. Además, la técnica de AI permitió disponer del tiempo en el aula para realizar la actividad de roles y discusión pública. Gracias a este espacio se pudo profundizar en el problema, empatizar con distintos puntos de vista e incluso pasar un rato agradable mejorando el ambiente y la cohesión del grupo.

El desarrollo y aplicación de todas las tecnologías conocidas por nuestro grupo de trabajo se han puesto de manifiesto en este trabajo. El próximo curso seguiremos con esta línea de investigación, ampliando el ámbito de estudio a otras competencias, profundizando más en las actividades más adecuadas para el desarrollo de cada una de ellas y planteando un estudio comparativo de las diferentes aplicaciones del Aula Inversa en los resultados de la adquisición de cada Competencia Transversal.

\section{Referencias}

[1] G. Moltó, J. F. Monserrat, I.C. Fita, and R. Picó, "Las Vídeo-Lecciones como Herramienta para la Adquisición Autónoma de Competencias Específicas en la Ingeniería" in Jornadas RED-U-Escuela Universitaria de Informática (EUI) UPM, 2013.

[2] Network, F. L. The four pillars of FLIPTM. www.flippedlearning.org/definition.

[3] G. Ion, E. Cano, E., \& Cabrera, "Competency Assessment Tool (CAT). The evaluation of an innovative competency-based assessment experience in higher education," Technology, Pedagogy and Education, 1-18, 2016.

[4] Proyecto de competencias transversales, UPV. http://competenciast.webs.upv.es

[5] Teo TW, Tan KCD, Yan YK, Teo YC, Yeo LW, "How flip teaching supports undergarudate chemistry laboratory learning", Chem. Educ. Res. Pract., 15, 550-567, 2014

[6] Segrelles, J. Damian, Germán Moltó, and Miguel Caballer. 2015. "Remote Computational Labs for Educational Activities via a Cloud Computing Platform." In 2015 Proceedings of the Information Systems Education Conference (ISECON), 309-21. http://proceedings.isecon.org/download/ki9hcpvocepkcwky5te9.

[7] Office Mix. https://mix.office.com/en-us/Home 